\title{
Feasibility of Optical Packet Switched WDM Networks without Packet Synchronisation Under Bursty Traffic Conditions
}

Fjelde, Tina; Hansen, Peter Bukhave; Kloch, Allan; Wolfson, David; Stubkjær, Kristian Elmholdt

\section{Published in:}

APCC/OECC '99. Fifth Asia-Pacific Conference on Communications and Fourth Optoelectronics and Communications Conference

Link to article, DOI:

10.1109/APCC.1999.824595

Publication date:

1999

Document Version

Publisher's PDF, also known as Version of record

Link back to DTU Orbit

Citation (APA):

Fjelde, T., Hansen, P. B., Kloch, A., Wolfson, D., \& Stubkjær, K. E. (1999). Feasibility of Optical Packet Switched WDM Networks without Packet Synchronisation Under Bursty Traffic Conditions. In APCC/OECC '99. Fifth AsiaPacific Conference on Communications and Fourth Optoelectronics and Communications Conference (Vol. 1, pp. 462-464). IEEE. https://doi.org/10.1109/APCC.1999.824595

\section{General rights}

Copyright and moral rights for the publications made accessible in the public portal are retained by the authors and/or other copyright owners and it is a condition of accessing publications that users recognise and abide by the legal requirements associated with these rights.

- Users may download and print one copy of any publication from the public portal for the purpose of private study or research.

- You may not further distribute the material or use it for any profit-making activity or commercial gain

- You may freely distribute the URL identifying the publication in the public portal 


\title{
Feasibility of optical packet switched WDM networks without packet synchronisation under bursty traffic conditions
}

\author{
T. Fjelde, P.B. Hansen, A. Kloch, D. Wolfson and K.E. Stubkjaer \\ Research Center COM \\ Technical University of Denmark, Building 349, DK-2800 Lyngby, Denmark \\ telephone: +45 45253797 , fax: +45459365 81, e-mail: tf@com.dtu.dk
}

\begin{abstract}
We show that complex packet synchronisation may be avoided in optical packet switched networks. Detailed traffic analysis demonstrates that packet loss ratios of $10^{-10}$ are feasible under bursty traffic conditions for a high capacity network consisting of asynchronously operated add-drop switch nodes with buffers having only $\sim 12$ fibre delay lines.
\end{abstract}

\section{Introduction}

Recently, there has been an increasing interest in the implementation of IP over optical WDM networks [1]. In consistence with this trend, optical packet switching seeks to combine packet switching with WDM techniques to yield a future proof optical platform for IP packet transmission. This solution is advantageous due to the high switching granularity and flexibility that optical packet switching offers.

When designing an optical packet switched network it is desirable to implement as many of the necessary functionalities as possible in the optical domain to ease the handling of large bandwidths. Unfortunately, the schemes proposed to perform optical packet synchronisation are, as yet, very complicated $[2,3]$. For that reason, it is of great interest to evaluate the traffic performance of optical WDM networks when the switch nodes are operated asynchronously (i.e., without synchronising the input packet stream to the node timing reference).

Previously, it has been shown that asynchronous packet switch operation is feasible with good traffic performance under the assumption of random traffic distribution [4]. In order to assess the feasibility of the asynchronous scheme it is, however, also necessary to:

(i.) Evaluate the overall traffic performance of the optical network.

(ii.) Assess the implications of different service types to the overall traffic performance. The assumption of random traffic distribution is inadequate, for example, in the case of IP/multimedia traffic, which is widely represented through a bursty traffic model [5].
In this paper we take the above points into account for the first time by evaluating the traffic performance of a network consisting of optical add-drop packet switch blocks using a bursty traffic model. We show that the increased network packet loss, that asynchronous operation of the switch blocks leads to, can be counteracted by using wavelength converters for contention resolution, thereby attaining a very good traffic performance. In this way, complex packet synchronisation units that degrade the signal quality are avoided.

\section{IP over optical packet switched network}

The expansion of the Internet is expected to increase the demand for capacity not only in the core network, but also in metropolitan area networks (MANs). Figure 1 shows a MAN architecture for the transport of IP traffic over a packet switched network. The network consists of interconnected add-drop packet switch nodes, where the add-drop ports are used to route traffic between the optical MAN and the underlying access network. In order to perform this, each add-inlet is connected to interworking units (IWUs) that generate optical packets by encapsulation of IP packets from the access network in the optical payload and perform proper header insertion. Equivalently, the drop-outlets are connected to IWUs that extract the IP packets from the optical packets before they are dropped to the access network. The optical packet switches are interconnected by network fibres, each carrying $\mathrm{N}$ wavelength channels, $\lambda_{1}, \ldots, \lambda_{\mathrm{N}}$.

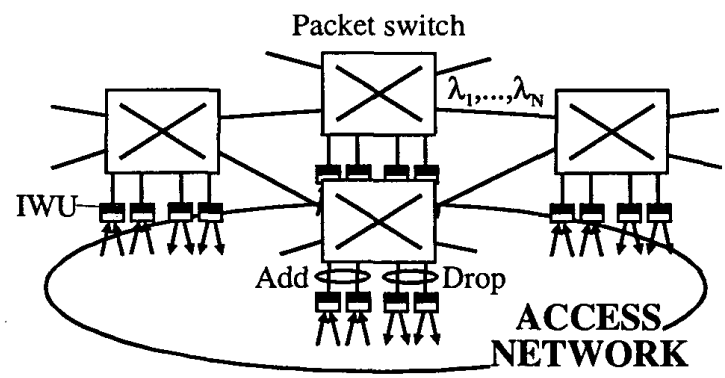

Fig. 1: Optical packet switched MAN architecture for transport of IP packets. 
An example of an optical add-drop packet switch block is shown in figure 2. It consists of $M$ network in- and outlets, $\mathrm{N}_{\mathrm{add}}$ add-inlets and drop-outlets with an electrical buffer. Each of the network fibres carries $\mathbf{N}$ wavelength channels. Tuneable wavelength converters are used to address free space in the optical buffers each consisting of $\mathrm{N}_{\mathrm{FDL}}$ fibre delay lines.

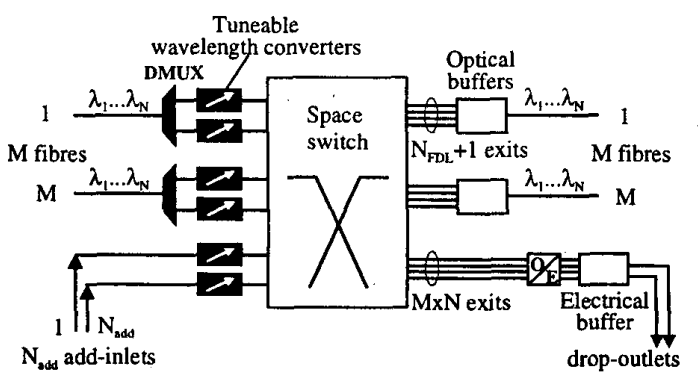

Fig. 2: Optical add-drop packet switch node with $M$ network in-and outlets and $N_{\text {add }}$ add-inlets. Each network in-and outlet carries $N$ wavelength channels.

This paper deals with the traffic performance of an adddrop network with asynchronously operated switch nodes, i.e. without packet alignment at the switch inlets. Asynchronous operation of the packet switch in figure 2 is possible because none of the included optical parts are inherently synchronous. The tuneable wavelength converters can be controlled with a variable offset to an internal clock, as can any active optical components (e.g., gates) within the space switch. Buffering by the fibre delay lines and the remaining multiplexers, splitters and waveguides are passive, thereby eliminating the need for complex optical packet synchronisation. Instead each packet will be tagged in time relative to a local clock by the electrical switch management. In this way, gates and converters can be controlled through a look-up table to accommodate the asynchronous operation.

\section{Traffic simulation model}

The traffic performance of an add-drop network is investigated using a general WDM Shufflenetwork with 32 add-drop switch nodes. The network consists of two columns with $164 \times 4$ nodes (M=4) in each (see [6] for further details on topology). The cylindrical connectivity pattern of the Shufflenetwork provides the capability of alternate routing in response to congestion and network failures [7] and ensures a small number of hops (switches) between the transmitting and receiving nodes, thus attaining a good traffic performance. Routing is performed by selecting the shortest path between source and destination node. If more than one path exists with an equal number of hops, a routing algorithm that minimises congestion is employed.

A computer simulation model has been developed to assess the traffic performance of the network when no packet alignment is performed. Packets are generated at the add-inlets, each inlet being modelled as a bursty traffic source. Furthermore, the electrical drop buffer is sufficiently large to ensure that the packet loss is not dominant. Placement of packets in the optical buffers is controlled to exploit the buffer capacity optimally. It is noted that the buffering and routing algorithm can violate packet sequence integrity because packets belonging to the same burst can be directed along different paths through the network. This can, however, be corrected at the interface to the access network, i.e. by the IWUs.

\section{Results}

Essential aspects concerning the performance of an asynchronously operated packet switched network under bursty traffic conditions are illustrated in figure 3 . The figure shows the packet loss ratio (PLR) for a 32-node Shufflenetwork as a function of the number of fibre delay lines when the load offered per add-inlet is 0.8 and the mean burst length is 4 . This relatively low level of burstiness is chosen because traffic shaping at the border between the packet switched layer and access layer reduces the burst length. Furthermore, the number of add-inlets to each node is equal to the number of wavelengths per network fibre $\left(\mathrm{N}_{\mathrm{add}}=\mathrm{N}\right)$. Only packet loss values down to $10^{-6}$ are shown, ensuring a reasonable computing time. Compared to synchronous switch operation, a significant increase in the PLR is observed for the asynchronous scheme because of a less efficient exploitation of buffer capacity. Employing 2 wavelengths per network fibre is, however, sufficient to compensate for this, while the total load offered by the two add-inlets is increased to $2 \times 0.8$. This improvement occurs because the use of WDM for contention resolution increases the flexibility of the delay line buffers. As seen in the figure, employing 4 wavelength channels per fibre lowers the packet loss significantly. Extrapolation predicts that a PLR of $\sim 10^{-10}$ is obtainable

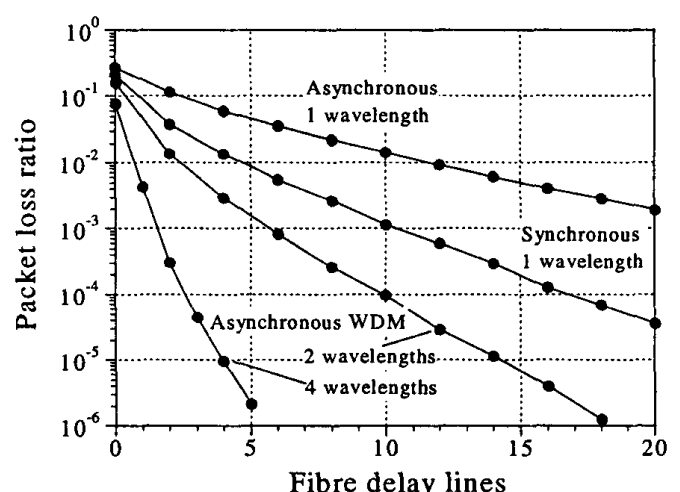

Fig. 3: Packet loss ratio for a 32-node Shufflenetwork with a load of 0.8 per add-inlet as a function of the number of fibre delay lines for both synchronous and asynchronous operation. The mean burst length is 4 . 
if the number of fibre delay lines is increased to $\sim 12$, while over 50 fibre delay lines are necessary in the synchronous case employing only one wavelength. This indicates that the requirement for commercial ATM networks of a PLR of $\sim 10^{-10}$ at a load of 0.8 is possible for asynchronous switch operation using a realistic number of fibre delay lines.

When employing a bursty traffic model to represent IP/multimedia traffic, the specific service type affects the mean burst length. Therefore, it is relevant to investigate the effect of burst length on PLR. As indicated in figure 3 , employing 4 wavelengths per fibre leads to a significant reduction in the packet loss ratio when the burst length is relatively low (i.e., 4). However, the use of WDM is also highly advantageous for larger values of mean burst length, as illustrated in figure 4 . The offered load per add-inlet is 0.8 and 8 fibre delay lines are assumed. It is observed that the mean burst length influences the PLR. However, the use of WDM significantly reduces the packet loss ratio. Employing 4 wavelengths per channel, as opposed to 2 per channel, results in a PLR that is reduced by two orders of magnitude almost independent of burst length.

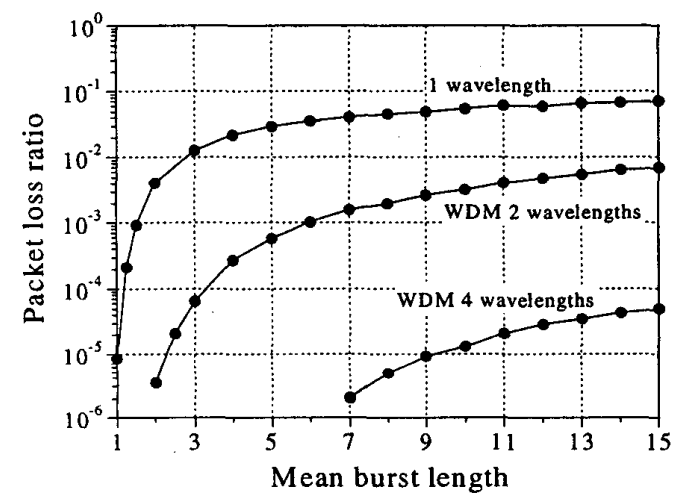

Fig. 4: Packet loss ratio for a 32-node Shufflenetwork operated asynchronously as a function of the mean burst length. The load per add-inlet is 0.8 and the number of fibre delay lines is 8.

\section{Conclusion}

In this paper, we have assessed the feasibility and performance of an optical add-drop network using asynchronously operated packet switches under bursty traffic conditions. An increase in packet loss ratio is observed for the asynchronous scheme compared to the synchronous case, which is, however, counteracted by the introduction of WDM. The requirement for commercial ATM networks of a PLR of $\sim 10^{-10}$ at a load of 0.8 is satisfied using 4 wavelength channels per fibre and $\sim 12$ fibre delay lines, while over 50 are necessary in the synchronous case using one wavelength Furthermore, it is indicated that asynchronous packet switching is feasible even under highly bursty traffic conditions through the use of WDM for contention resolution. Thus, optical networks operated asynchronously are rendered highly competitive by eliminating the need for complex optical packet synchronisation.

\section{References}

[1] C. Guillemot et al., ECOC'98, Vol. 1, pp. 83-85, Madrid, Spain, Sept. 1998.

[2] M. Burzio et al., Photonics in Switching'95, Vol. 12, pp. 64-66, Salt Lake City, U.S.A., March 1995.

[3] D. Chiaroni et al., ECOC'97, Vol. 3, pp. 148-151, Edinburgh, Scotland, Sept. 1997.

[4] P.B. Hansen et al., ECOC'98, Vol. 1, pp. 591-592, Madrid, Spain, Sept. 1998.

[5] I.W. Habib, IEEE Communications Magazine, Vol. 30, Is. 7, pp. 48-54, July 1992.

[6] A.S. Acampora, IEEE GLOBECOM'87, pp. 1459 1467,1987 .

[7] A.S. Acampora et al., IEEE Network, Vol. 3, Is. 1, pp. 29-41, Jan. 1989. 